Review Article

\title{
A Comparison of Chemotherapy Used with and without Apatinib for Patients with Ovarian Carcinoma Who Progressed after Standard Regimens: A Systematic Review and Meta-Analysis
}

\author{
Chao Hou $\mathbb{D}^{1,2}$ Zhuang-Zhuang Jiang, ${ }^{1,2}$ Bo Pan, ${ }^{1,2}$ Xiao-Chun Zhang $\mathbb{D}^{3}{ }^{3}$ \\ and Yan-Qing Liu $\mathbb{D}^{1,2}$ \\ ${ }^{1}$ College of Medicine, Yangzhou University, Yangzhou 225001, Jiangsu, China \\ ${ }^{2}$ The Key Laboratory of Syndrome Differentiation and Treatment of Gastric Cancer of the State \\ Administration of Traditional Chinese Medicine, Yangzhou 225001, China \\ ${ }^{3}$ College of Medicine, Yangzhou Hospital of Traditional Chinese Medicine Affliated to Nanjing University of Chinese Medicine, \\ Yangzhou 225009, Jiangsu, China
}

Correspondence should be addressed to Xiao-Chun Zhang; ceiq@sina.com and Yan-Qing Liu; liuyq@yzu.edu.cn

Received 9 May 2021; Accepted 16 October 2021; Published 3 November 2021

Academic Editor: Juntra Karbwang

Copyright (c) 2021 Chao Hou et al. This is an open access article distributed under the Creative Commons Attribution License, which permits unrestricted use, distribution, and reproduction in any medium, provided the original work is properly cited.

Objective. This meta-analysis was conducted to compare the therapeutic efficacy and clinical safety of the combination therapy of apatinib plus chemotherapy with that of chemotherapy alone in patients with refractory or recurrent ovarian carcinoma (OC). Methods. Relevant randomized controlled trials (RCT) or case-control studies (CCS) were identified by searching Chinese and English databases up to October 31, 2020. The risk of methodological bias tool and Newcastle-Ottawa scale (NOS) were used to assess trial quality. Pooled odds ratios (OR) and 95\% confidence intervals (CI) were calculated to evaluate the therapeutic effects and adverse drug reactions. Subgroup analyses of study type, study sample size, dosage of apatinib, and chemotherapy regimen between treatment group and control group were performed. Publication bias was assessed by funnel plot symmetry, Begg-Mazumdar test, and Egger test. The robustness of our results was presented by removing the trial one by one. Results. Fifteen eligible studies covering 1,020 patients were included in this review and meta-analysis. Among these studies, 8 were RCTs, and 7 were CCSs. Compared with chemotherapy alone, apatinib plus chemotherapy significantly increased objective response rate $(\mathrm{OR}=3.55 ; 95 \% \mathrm{CI} 2.31$ to 5.47$)$, disease control rate $(\mathrm{OR}=3.04 ; 95 \% \mathrm{CI} 2.12$ to 4.36$)$, and overall survival $(\mathrm{OR}=5.03 ; 95 \% \mathrm{CI} 3.16$ to 6.90). Conclusions. The combination treatment of apatinib plus chemotherapy provides better clinical benefits for OC patients when compared to chemotherapy alone and should be recommended for suitable patients with OC after the failure of standard regimens. However, further investigation into future large-scale prospective randomized research is still needed.

\section{Introduction}

Ovarian carcinoma (OC) is one of the common malignant carcinomas in women worldwide. In 2018 alone, over 295,000 women developed OC, of whom over 184,000 died globally [1]. Although surgery and chemotherapy can be curative for patients with early-stage OC, most women with advanced OC suffer repeated recurrences of the disease with a progressively shorter disease-free interval [2] and platinum resistance [3]. Unfortunately, there are few interventions that can postpone or stop the malignant course of the illness for patients who progressed after standard regimens. As no apparent breakthrough in the treatment of OC over the past two decades, the five-year overall survival rate is still $<30 \%$ for patients with advanced-stage OC [4].

Angiogenesis plays an important role in the oncogenesis, development, and metastasis of OC [5]. Apatinib, as an inhibitor of vascular endothelial growth factor receptor 2 , is 
widely used in China as an antiangiogenic drug. Studies have confirmed the antitumor activity of apatinib in the treatment of lung cancer [6], liver cancer [7], and other malignant tumors. For OC, increasing clinical studies have confirmed that apatinib alone or apatinib combined with chemotherapy can prolong the progression-free survival (PFS) of recurrent $\mathrm{OC}$ that is resistant to platinum-based chemotherapeutics [8-10]. However, due to the small sample size of relevant clinical studies and a lack of multicenter randomized controlled clinical trials, there is still a lack of sufficient evidence to confirm the clinical efficacy and safety of apatinib combined with chemotherapy in the treatment of OC. Therefore, we have performed a systematic review and meta-analysis to assess the efficacy and safety of apatinib plus chemotherapy.

\section{Materials and Methods}

2.1. Inclusion Criteria. The systematic review and metaanalysis were conducted according to the Preferred Reporting Items for Systematic Reviews and Meta-Analyses (PRISMA) guidelines. The inclusion criteria for the studies were as follows: (1) the type of included study was a randomized controlled trial (RCT) or case-control study (CCS), and there was no limit to follow-up procedures or type of setting; (2) the participants were confirmed to have OC pathologically or computed tomography, and there was no restriction on gender, race, or nationality for them; and (3) the intervention was apatinib combined with chemotherapy versus chemotherapy alone, and no patients had received chemotherapy, radiotherapy, targeted therapy, or other therapies within one month before treatment.

2.2. Exclusion Criteria. We excluded studies with any of the following characteristics: (1) the study was neither RCT nor CCS and involved nonovarian carcinoma, nonapatinib, or apatinib alone; (2) the patients were not confirmed to have OC; (3) the control measures did not include chemotherapy but other therapies such as operation or radiotherapy; (4) the data could not be extracted or were repeated; (5) the study was a review or unrelated meta-analyses, animal study, case report, conference abstracts, or letters to journal editors; and (6) studies had no data on tumor response, tumor-associated antigen (TAA), survival, and adverse drug reaction (ADR).

2.3. Retrieval Strategy. The retrieval strategy was built using $\mathrm{MeSH}$ and free words. The retrieval form was (apatinib OR rivoceranib esylate OR YN968D1 OR YN-968D1 OR rivoceranib OR apatinib mesylate) AND (“Ovarian Neoplasms" [Mesh] OR Neoplasm, Ovarian OR Ovarian Neoplasm OR Ovary Neoplasms OR Neoplasm, Ovary OR Neoplasms, Ovary OR Ovary Neoplasm OR Neoplasms, Ovarian OR Ovary Cancer OR Cancer, Ovary OR Cancers, Ovary OR Ovary Cancers OR Ovarian Cancer OR Cancer, Ovarian OR Cancers, Ovarian OR Ovarian Cancers OR Cancer of Ovary OR Cancer of the Ovary). Two investigators independently retrieved all related studies from the following databases: China Biological Medicine Database (CBM), China National
Knowledge Infrastructure Database (CNKI), Chinese Scientific Journals Full-Text Database (VIP), Wanfang Data, Cochrane Library, PubMed, Greenmedical, and EBSCO (up to October 2020). In addition, we evaluated all related systematic reviews or meta-analyses and selected eligible studies from their references.

2.4. Study Selection. According to the predefined inclusion and exclusion criteria, two investigators independently selected eligible studies. Any disagreements were resolved by discussion between these two or with a third investigator.

2.5. Methodological Quality Assessment. The methodological quality of the included RCTs was assessed using the risk of bias tool of the Review Manager software 5.3. The criteria for assessment of the risk of bias included specific methods of randomization and allocation concealment, the blinding method, and reporting of dropouts or withdrawal of patients and other bias according to the Cochrane Evaluation Handbook for Systematic Reviews of Interventions Version 5.3. We judged each item as "yes" for a low risk of bias, "no" for high risk, and "unclear" otherwise. Two investigators independently assessed the risk of methodological bias of all included studies. Any disagreements about the decisions of high, low, or unclear risk were resolved by discussion with each other or a third investigator. Similarly, two investigators independently evaluated the quality of the included CCSs by Newcastle-Ottawa scale (NOS). Any disagreements about the decisions of the score were resolved by discussion with each other or with a third investigator.

2.6. Outcome Definition. Primary outcomes were tumor response, TAA, and survival. The outcomes for tumor response were the objective response rate (ORR) and disease control rate (DCR) according to the World Health Organization (WHO) guidelines for solid tumor responses or Response Evaluation Criteria in Solid Tumors (RECIST) [11]. The indicators used were complete response (CR), partial response (PR), no change (NC), and progressive disease (PD). The ORR was equal to $\mathrm{CR}$ plus $\mathrm{PR}$, and the DCR was equal to CR plus PR and NC. The survival was assessed using overall survival (OS), time to progression (TTP), and PFS. Secondary outcomes were ADRs, which were identified according to the WHO standards [12] or Common Terminology Criteria for Adverse Events (CTCAE) [13].

2.7. Data Extraction. Three researchers independently extracted the following information from the included RCTs and CCSs: the first author's name, time of publication, treatment process, age, gender, sample size, dose of apatinib, chemotherapy regimen, and outcomes including ORR, DCR, OS, TTP, PFS, TAA, and ADRs. For crossover studies, only data from the first portion of the study would have been incorporated to avoid possible carryover effects of medications into the second part of the study and make these studies more comparable to those studies not of crossover 
design. If the reports were sufficiently detailed, the data were extracted directly from the papers. Otherwise, we contacted the authors for further information. When no author replied, the data were reconstructed using a software graph digitizer scout according to the graphed data in the paper $[14,15]$.

2.8. Statistical Analysis. Meta-analysis was implemented by two reviewers using Review Manager 5.3 (as recommended by the Cochrane Collaboration). The odds ratios (OR) and 95\% confidence interval (CI) were calculated, and $P<0.05$ was considered statistically significant. Statistical heterogeneity of the results among the studies was assessed by Cochran's $\chi^{2}$ test and the $I^{2}$ statistic, and the inconsistency was calculated by $I^{2}$. When $I^{2} \leq 50 \%$ and $P>0.1$, there was no statistical heterogeneity among studies; if $I^{2}>50 \%$ and $P<0.1$, there was statistical heterogeneity among studies. When heterogeneity was confirmed, the random-effects model (REM) was used. Otherwise, a fixed-effects model (FEM) was used. If $I^{2}>50 \%$ and with good clinical consistency, we adopted a random-effects model (REM). Otherwise, we discarded the outcome. Subgroup analyses were performed according to apatinib alone or plus different chemotherapy therapies and revealed their influence on the clinical effect of apatinib combined with chemotherapy. Furthermore, publication bias was assessed by funnel plot symmetry, the Egger test, and the Begg test with $P<0.05$ suggesting obvious publication bias [16].

According to recent guidance [17], we established a subgroup analysis model to examine heterogeneity. Moreover, subgroup analysis was performed according to study type, study sample size, treatment process, dose of apatinib, chemotherapy regimen, and cycle aimed to reveal their influence on the clinical efficacy of apatinib combined with chemotherapy. We conducted a univariate metaregression for the relationship between each variable and tumor response. We also conducted multiple regression analysis, adjusting for the OR of tumor response at baseline, dose of apatinib, and different chemotherapy regimens.

We chose the STATA 11.0 software (STATA Corporation, College Station, TX) as a graphical tool to present the results' robustness by removing the trials one by one.

\section{Results}

3.1. Search Results. Following the process of the PRISMA flow diagram in Figure 1, 315 potentially relevant publications were identified. All records were imported into EndNote X6. Two reviewers screened all records using a three-step process. First, we screened the titles, excluding the duplicate including 195 records. Second, we read the abstracts and excluded irrelevant studies $(n=48)$, comments $(n=3)$, reviews $(n=7)$, case reports $(n=13)$, and clinical registrations $(n=11)$. Third, we assessed the full texts and excluded studies with unavailable data $(n=8)$ and singlearm studies $(n=15)$. Finally, we included 15 studies including 7 CCSs and 8 RCTs (Figure 1 and Table 1).
3.2. Characteristics of the Included Trials. This meta-analysis involved 15 studies containing 1,020 ovarian carcinoma patients who progressed after standard regimens in China. Combined administration with apatinib and chemotherapy was administered in 504 cases, and 516 cases were administered chemotherapy alone. Detailed information of the 15 studies is presented in Table 1.

3.3. Methodological Quality Assessment. The included RCTs underwent a quality assessment using the risk of bias tool of the Review Manager software 5.3, and the outcome is shown in Figure 2. The quality of the included case-control studies was assessed by NOS, and the outcome is shown in Table 2.

3.4. Objective Response Rate. Cochran's $\chi^{2}$ test showed statistical heterogeneity for ORR $\left(P=0.09 ; I^{2}=15 \%\right)$. Therefore, we synthesized the data using an REM. Compared with chemotherapy alone, the results of the meta-analysis showed that apatinib in combination with chemotherapy significantly increased ORR $(\mathrm{OR}=3.55 ; 95 \%$ CI 2.31 to 5.47; $P<0.00001$; Figure 3).

3.5. Disease Control Rate. Cochran's $\chi^{2}$ test and $I^{2}$ statistic showed minimal heterogeneity for DCR $\left(P=0.30 ; I^{2}=15 \%\right)$. Therefore, we synthesized the data using an FEM. Compared with chemotherapy alone, the results of the meta-analysis showed that apatinib plus chemotherapy significantly increased DCR $(\mathrm{OR}=3.04 ; 95 \%$ CI 2.12 to 4.36 ; $P<0.00001$; Figure 4).

3.6. Overall Survival. Cochran's $\chi^{2}$ test and $I^{2}$ statistic showed obvious heterogeneity for OS $(P<0.00001$; $\left.I^{2}=98 \%\right)$. Therefore, we synthesized the data using an REM. The results demonstrated that apatinib plus chemotherapy significantly increased $\mathrm{OS}(\mathrm{OR}=5.03 ; 95 \% \mathrm{CI} 3.16$ to 6.90 ; $P<0.00001$; Figure 5).

3.7. Levels of Tumor-Associated Antigen. Cochran's $\chi^{2}$ test and $I^{2}$ statistics showed statistical heterogeneity for CA125 $\left(P<0.00001 ; I^{2}=98 \%\right)$ and no heterogeneity for CEA $\left(P=0.85 ; I^{2}=0 \%\right)$. Thus, we synthesized the data of CA125 and CEA using REM and FEM, respectively. The results of the meta-analysis demonstrated that apatinib in combination with chemotherapy significantly decreased the level of CEA $(\mathrm{MD}=-4.65 ; 95 \%$ CI -5.52 to $-3.77 ; P<0.00001$; Figure 6), although did not significantly decreased the level of CA125 $(\mathrm{MD}=-36.91 ; 95 \% \mathrm{CI}-88.39$ to $14.57 ; P=0.16$; Figure 7).

3.8. Adverse Drug Reactions. Cochran's $\chi^{2}$ test and $I^{2}$ statistics showed statistical heterogeneity for hypertension $\left(P=0.02 ; I^{2}=53 \%\right)$ and hand-foot syndrome $(P=0.04$; $\left.I^{2}=55 \%\right)$; minimal heterogeneity for proteinuria $\left(I^{2}=30 \%\right)$; and no heterogeneity $\left(P>0.1 ; I^{2}=0 \%\right)$ for myelosuppression, leucopenia, gastrointestinal reaction, nausea/ vomiting, liver/renal dysfunction, and fatigue. Therefore, we 

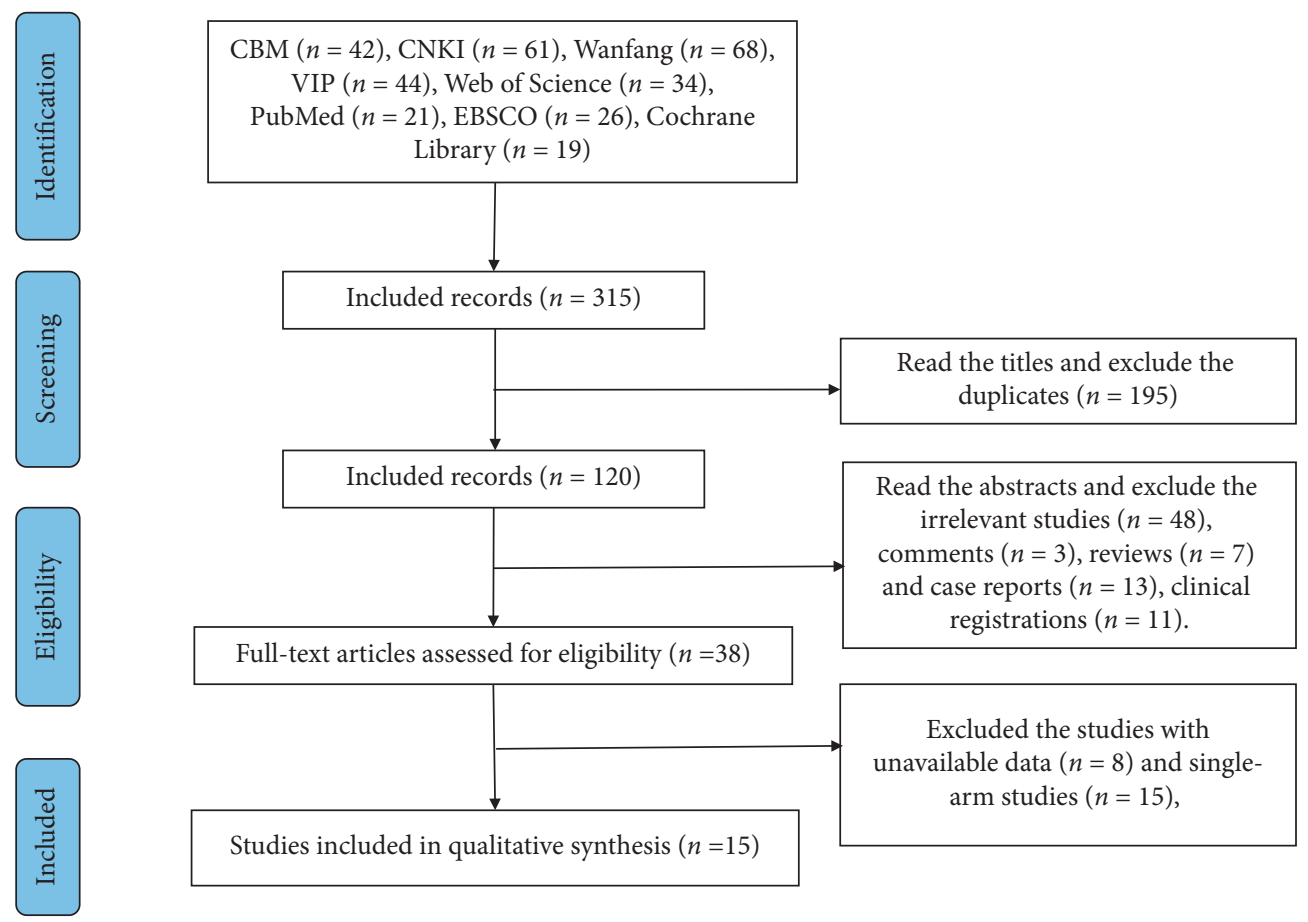

Figure 1: Articles retrieved and assessed for eligibility.

TABLE 1: Characteristics of the included trials.

\begin{tabular}{|c|c|c|c|c|c|c|c|c|}
\hline \multirow{2}{*}{$\begin{array}{l}\text { Trial } \\
\text { type }\end{array}$} & \multirow{2}{*}{$\begin{array}{l}\text { First author, } \\
\text { year }\end{array}$} & \multicolumn{3}{|c|}{ Patient } & \multirow{2}{*}{$\begin{array}{l}\text { Apatinib } \\
\left(\mathrm{mg}^{-1} \mathrm{~d}^{-1}\right)\end{array}$} & \multirow{2}{*}{$\begin{array}{c}\text { Intervention (regimen, cycle, or } \\
\text { duration) }\end{array}$} & \multirow{2}{*}{ Criteria } & \multirow{2}{*}{ Outcomes } \\
\hline & & Age (years) & No. $(\mathrm{E} / \mathrm{C})$ & $\mathrm{TP}$ & & & & \\
\hline CCS & $\mathrm{Li}, 2018$ [18] & $26-70^{\mathrm{a}}$ & $21 / 25^{\mathrm{d}}$ & FSMC & 500 & $\begin{array}{l}\text { E: apatinib + taxane or anthracycline, } \\
\quad 6 \text { cycles } \\
\text { C: taxane or anthracycline, } 6 \text { cycles }\end{array}$ & $\begin{array}{l}\text { RECIST } \\
1.1 \\
\text { CTCAE }\end{array}$ & $\mathrm{O} 1,4$ \\
\hline CCS & $\begin{array}{l}\text { Cheng et al., } \\
2018 \text { [19] }\end{array}$ & Un & $46 / 46^{\mathrm{d}}$ & FSMC & 250 & $\begin{array}{c}\text { E: apatinib }+ \text { taxanes, } 6 \text { cycles } \\
\text { C: taxane, } 6 \text { cycles }\end{array}$ & $\begin{array}{l}\text { Un, } \\
\text { CTCAE }\end{array}$ & $\mathrm{O} 1,4$ \\
\hline CCS & $\begin{array}{l}\text { Shao et al., } \\
2019 \text { [20] }\end{array}$ & $\begin{array}{c}64.2 \pm 1.63 / \\
58.55 \pm 1.45^{\mathrm{b}}\end{array}$ & $54 / 54^{\mathrm{d}}$ & Un & 500 & $\begin{array}{l}\text { E: apatinib + taxane + platinum, Un } \\
\text { C: taxane + platinum, Un }\end{array}$ & Un, Un & O1. 4 \\
\hline CCS & $\begin{array}{l}\text { Liu et al., } \\
2020[21]\end{array}$ & $\begin{array}{l}36.9 \pm 5.1 / \\
45.1 \pm 4.9^{\mathrm{b}}\end{array}$ & $22 / 22^{\mathrm{d}}$ & FSMC & 500 & $\begin{array}{c}\text { E: apatinib + taxanes or } \\
\text { anthracycline, Un } \\
\text { C: taxanes or anthracycline, Un }\end{array}$ & $\begin{array}{l}\text { RECIST } \\
1.1, \mathrm{WHO}\end{array}$ & $\mathrm{O} 1,4$ \\
\hline CCS & $\begin{array}{l}\text { Zhang and } \\
\text { Shi, 2020 } \\
\text { [22] }\end{array}$ & $18-70^{\mathrm{a}}$ & $21 / 25^{\mathrm{d}}$ & FPC & 500 & $\begin{array}{l}\text { E: apatinib + gemcitabine, Un } \\
\text { C: gemcitabine, Un }\end{array}$ & $\begin{array}{l}\text { RECIST } \\
1.1, \text { Un }\end{array}$ & $\mathrm{O} 1,3,4$ \\
\hline CCS & $\begin{array}{l}\text { Zhang and } \\
\text { Xiong, } 2019 \\
{[23]}\end{array}$ & $\begin{array}{l}53.4 \pm 4.2 / \\
53.3 \pm 4.6^{\mathrm{b}}\end{array}$ & $30 / 30^{\mathrm{d}}$ & Un & 500 & $\begin{array}{l}\text { E: apatinib + platinum, } 8 \text { cycles } \\
\text { C: platinum, } 8 \text { cycles }\end{array}$ & Un & $\mathrm{O} 4$ \\
\hline CCS & $\begin{array}{c}\text { Zhao, } 2018 \\
\quad[24]\end{array}$ & $49-75^{\mathrm{a}}$ & $16 / 20^{\mathrm{d}}$ & FPC & 500 & $\begin{array}{c}\text { E: apatinib + anthracycline, } 6 \text { cycles } \\
\text { C: anthracycline, } 6 \text { cycles }\end{array}$ & $\begin{array}{l}\text { RECIST } \\
1.1, \text { Un }\end{array}$ & $\mathrm{O} 1,4$ \\
\hline RCT & $\begin{array}{l}\text { Feng et al., } \\
2019 \text { [25] }\end{array}$ & $\begin{array}{l}45.8 \pm 5.7 / \\
46.2 \pm 6.1^{\mathrm{b}}\end{array}$ & $30 / 30^{\mathrm{d}}$ & FPC & Un & $\begin{array}{l}\text { E: apatinib + platinum, } 4 \text { cycles } \\
\text { C: platinum, } 4 \text { cycles }\end{array}$ & Un, Un & $\mathrm{O} 1,4$ \\
\hline RCT & $\begin{array}{l}\text { Ren, } 2019 \\
\text { [26] }\end{array}$ & $\begin{array}{l}74.0 \pm 1.3 / \\
75.0 \pm 1.5^{\mathrm{b}}\end{array}$ & $38 / 38^{\mathrm{d}}$ & FSMC & 500 & $\begin{array}{l}\text { E: apatinib + platinum, Un } \\
\text { C: platinum, Un }\end{array}$ & WHO, Un & $\mathrm{O} 1,2,3,4$ \\
\hline RCT & $\begin{array}{l}\text { Wang and } \\
\text { Qu, 2019 } \\
\text { [27] }\end{array}$ & $50.68 \pm 16.84^{C}$ & $39 / 39^{\mathrm{d}}$ & FPC & 425 & $\begin{array}{c}\text { E: apatinib }+ \text { taxane }+ \text { platinum, } 3 \\
\text { cycles } \\
\text { C: taxane + platinum, } 3 \text { cycles }\end{array}$ & $\begin{array}{l}\text { RECIST } \\
1.1, \text { Un }\end{array}$ & $\mathrm{O} 1,3,4$ \\
\hline RCT & $\begin{array}{l}\text { Liu, } 2015 \\
\quad[28]\end{array}$ & $\begin{array}{l}48.5 \pm 5.5 / \\
48.0 \pm 5.0^{b}\end{array}$ & $37 / 37^{\mathrm{d}}$ & Un & 850 & $\begin{array}{c}\text { E: apatinib + taxane + platinum, } 4 \\
\text { cycles } \\
\text { C: taxane + platinum, } 4 \text { cycles }\end{array}$ & WHO & $\mathrm{O} 4$ \\
\hline
\end{tabular}


TABLE 1: Continued.

\begin{tabular}{|c|c|c|c|c|c|c|c|c|}
\hline \multirow{2}{*}{$\begin{array}{l}\text { Trial } \\
\text { type }\end{array}$} & \multirow{2}{*}{$\begin{array}{l}\text { First author, } \\
\text { year }\end{array}$} & \multicolumn{3}{|c|}{ Patient } & \multirow{2}{*}{$\begin{array}{l}\text { Apatinib } \\
\left(\mathrm{mg} \cdot \mathrm{d}^{-1}\right)\end{array}$} & \multirow{2}{*}{$\begin{array}{l}\text { Intervention (regimen, cycle, or } \\
\text { duration) }\end{array}$} & \multirow{2}{*}{ Criteria } & \multirow{2}{*}{ Outcomes } \\
\hline & & Age (years) & No. (E/C) & $\mathrm{TP}$ & & & & \\
\hline RCT & $\begin{array}{l}\text { Zhao et al., } \\
2019 \text { [29] }\end{array}$ & $\begin{array}{l}54.29 \pm 6.87 / \\
54.76 \pm 6.72^{\mathrm{b}}\end{array}$ & $19 / 19^{\mathrm{d}}$ & FSMC & 500 & $\begin{array}{l}\text { E: apatinib + taxane or anthracycline, } \\
\text { Un } \\
\text { C: taxane or anthracycline, Un }\end{array}$ & Un & O1 \\
\hline RCT & $\begin{array}{l}\text { Zheng et al., } \\
2019 \text { [30] }\end{array}$ & $\begin{array}{l}60.5 \pm 5.1 / \\
60.1 \pm 4.9^{\mathrm{b}}\end{array}$ & $40 / 40^{\mathrm{d}}$ & FPC & Un & $\begin{array}{l}\text { E: apatinib }+ \text { taxane }+ \text { anthracycline, } \\
\quad 6-8 \text { cycles } \\
\text { C: taxane + anthracycline, } 6-8 \text { cycles }\end{array}$ & WHO, Un & $\mathrm{O} 1,4$ \\
\hline RCT & $\begin{array}{l}\text { Quan, } 2020 \\
\text { [31] }\end{array}$ & $\begin{array}{l}55.89 \pm 7.76 / \\
55.21 \pm 7.71^{\mathrm{b}}\end{array}$ & $50 / 50^{\mathrm{d}}$ & FPC & 250 & $\begin{array}{l}\text { E: apatinib + taxane or anthracycline, } \\
\text { Un } \\
\text { C: taxane or anthracycline, Un }\end{array}$ & Un, Un & $\mathrm{O} 1,2,4$ \\
\hline RCT & $\begin{array}{l}\text { Ran and Liu, } \\
2020[32]\end{array}$ & $\begin{array}{l}52.10 \pm 12.19 / \\
51.37 \pm 12.53^{b}\end{array}$ & $41 / 41^{\mathrm{d}}$ & FFC & 500 & $\begin{array}{c}\text { E: apatinib + gemcitabine, } 3 \text { cycles } \\
\text { C: gemcitabine, } 3 \text { cycles }\end{array}$ & $\begin{array}{l}\text { RECIST } \\
1.1 \\
\text { CTCAE }\end{array}$ & $\mathrm{O} 1,2,3,4$ \\
\hline
\end{tabular}

Note: CCS: case-control study; RCT: randomized clinical trial; E: experimental group; C: control group; Un: unclear; ${ }^{a}$ all patients (range); ${ }^{\mathrm{b}} \mathrm{E} / \mathrm{C}(\mathrm{mean} \pm \mathrm{SD})$; ${ }^{C}$ all patients $($ mean $\pm S D) ;{ }^{d}$ the number of patients in experimental and control groups; TP: treatment process; FFC: failure of first-line chemotherapy; FPC: failure of platinum-based chemotherapy; FSMC: failure of second-line or multiple-line chemotherapy; WHO: World Health Organization guidelines for solid tumor responses, adverse events, and quality of life; RECIST 1.1: Version 1.1 of Response Evaluation Criteria in Solid Tumors; CTCAE: Common Terminology Criteria for Adverse Events; O: outcomes; O1: tumor response including objective response rate and disease control rate; O2: survival status including overall survival, time to progression, and progression-free survival; O3: tumor-associated antigen; and O4: adverse drug reactions.

synthesized the OR of hypertension and hand-foot syndrome using an REM and of other ADRs data using an FEM. The results of the meta-analysis demonstrated that apatinib in combination with chemotherapy resulted in a higher risk of hypertension $(\mathrm{OR}=3.18 ; 95 \% \mathrm{CI} 1.63$ to $6.20 ; P<0.001)$, proteinuria $(\mathrm{OR}=3.14 ; 95 \% \mathrm{CI} 1.51$ to $6.52 ; P=0.002)$, and hand-foot syndrome $(\mathrm{OR}=4.39 ; 95 \% \mathrm{CI} 1.59$ to 12.15 ; $P=0.004)$. No statistical differences were shown in myelosuppression, leucopenia, gastrointestinal reaction, nausea/ vomiting, liver/renal dysfunction, and fatigue between the two groups (Table 3 and Figures S1-S9).

3.9. Subgroup and Metaregression Analysis. Subgroup analyses of study type, dosage of apatinib, study sample size, treatment process, dosage of apatinib, chemotherapy regimen, and cycle between treatment and control groups were performed (Table 4 and Figures S10-S21).

3.9.1. Study Type. Randomized clinical trials and casecontrol studies: subgroup analysis demonstrated that the heterogeneity $\left(I^{2}=62 \% ; P=0.01\right)$ of RCTs in ORR was significant. Multiple metaregression analysis also found statistical significance in the correlation between the study type and DCR $(P=0.043)$. Therefore, the heterogeneity of the studies in ORR and DCR might be from the RCTs.

3.9.2. Study Sample Size. Fifty patients or more versus less than 50 patients: subgroup analysis demonstrated that there was a significant difference between the subgroups with different sample sizes in heterogeneity. Univariate metaregression analysis found statistical significance in the correlation between the study sample size and ORR $(P=0.029)$. Additionally, univariate and multiple metaregression both found statistical significance in the correlation between the study sample size and DCR $(P=0.018$ and 0.030 , respectively). Thus, the study sample size might be the other source of the heterogeneity of ORR and DCR.

3.9.3. Treatment Process. Failure of first-line chemotherapy (FFC), failure of platinum-based chemotherapy (FPC), and failure of second- or multiple-line chemotherapy (FSMC): subgroup analysis demonstrated that the heterogeneity ( $I=55 \% ; P=0.07)$ of the subgroup with patients suffering FSMC was more obvious than other subgroups in ORR, indicating the subgroup might increase the overall heterogeneity in ORR. Additionally, subgroup analyses showed that patients receiving apatinib plus chemotherapy who suffered FSMC had significantly better ORR $(\mathrm{OR}=6.00 ; 95 \%$ CI 2.31 to $15.58 ; P<0.001)$ and DCR $(\mathrm{OR}=3.75 ; 95 \% \mathrm{CI} 2.19$ to $6.42 ; P<0.001)$.

3.9.4. Dosage of Apatinib. A dosage of $250,425,500$, and unclear $\mathrm{mg} / \mathrm{d}$. Subgroup analysis demonstrated that patients receiving apatinib plus chemotherapy who were administrated with apatinib of $500 \mathrm{mg} / \mathrm{d}$ had significantly better ORR $(\mathrm{OR}=4.10 ; 95 \%$ CI $2.10,8.00 ; P<0.001)$ and DCR $(\mathrm{OR}=3.08 ; 95 \%$ CI $1.98,4.81 ; P<0.001)$.

3.9.5. Chemotherapy Regimen. Platinum, taxane or anthracycline, taxane + platinum, gemcitabine, and taxane + anthracycline: subgroup analysis demonstrated that the heterogeneity of the subgroup with patients suffering chemotherapy based on taxane or anthracycline was obvious in ORR $\left(I^{2}=58 \% ; P=0.04\right)$ and DCR $\left(I^{2}=43 \% ; P=0.13\right)$, indicating the subgroup might increase the overall heterogeneity in ORR and DCR. Additionally, the heterogeneity of the subgroup with patients suffering chemotherapy based on taxane plus platinum was more obvious than those of other subgroups in DCR $\left(I^{2}=62 \% ; P=0.10\right)$, indicating the subgroup might also increase the overall heterogeneity in DCR. Subgroup analyses also showed that patients receiving 


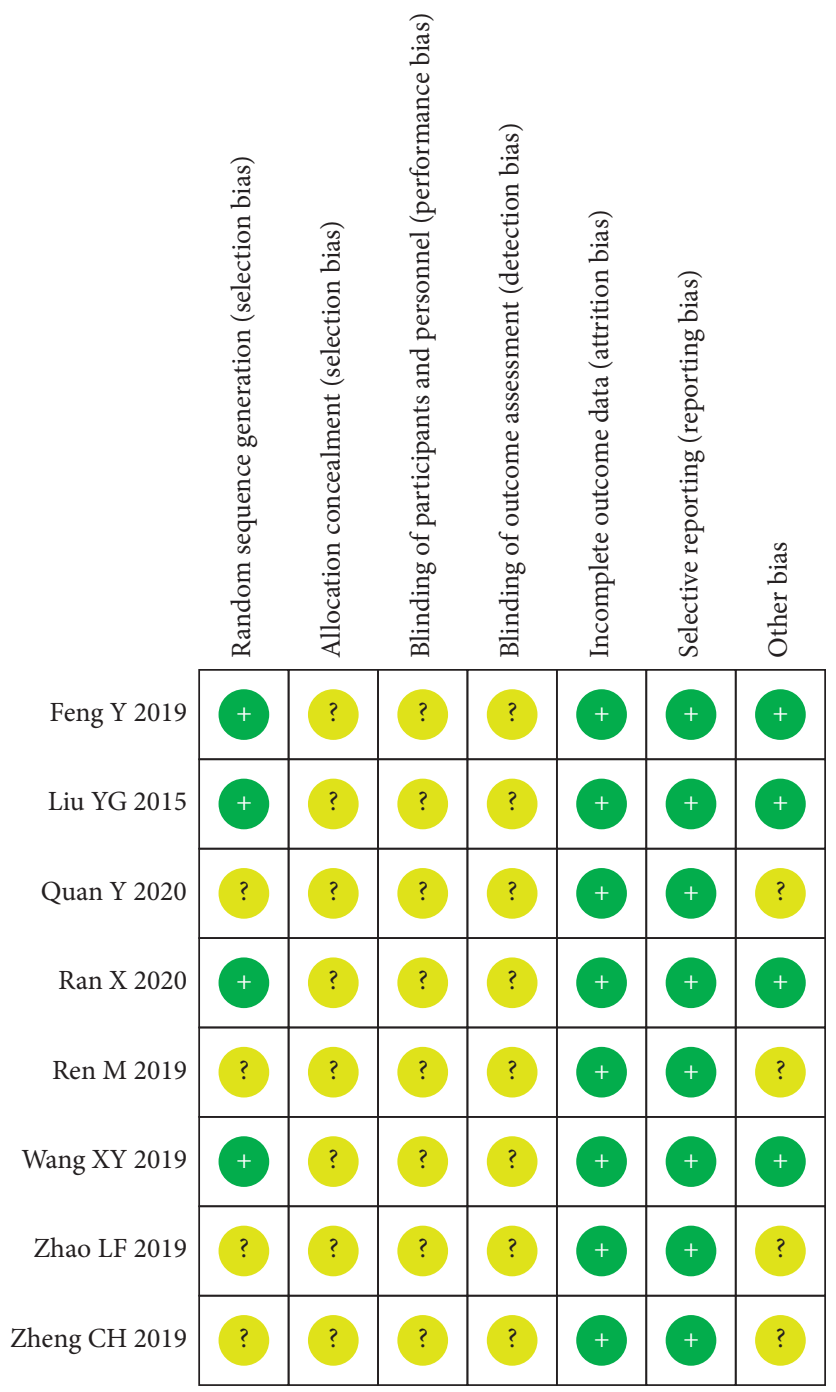

(a)

Random sequence generation (selection bias) Allocation concealment (selection bias)

Blinding of participants and personnel (performance bias)

Blinding of outcome assessment (detection bias)

Incomplete outcome data (attrition bias)

Selective reporting (reporting bias)

Other bias

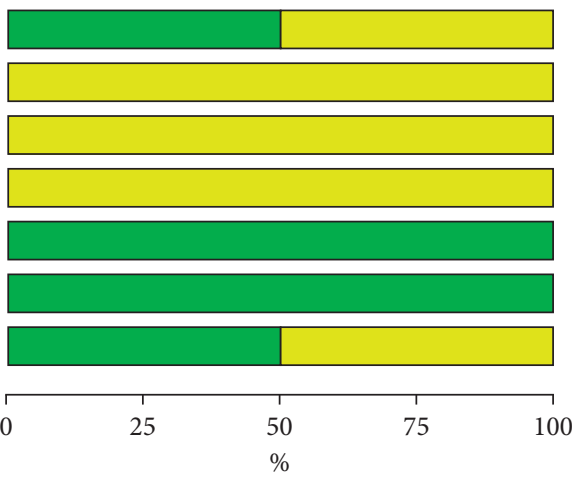

Low risk of bias

$\square$ Unclear risk of bias

High risk of bias

(b)

FIGURE 2: The risk of methodological bias: (a) risk of bias summary and (b) risk of bias graph. 


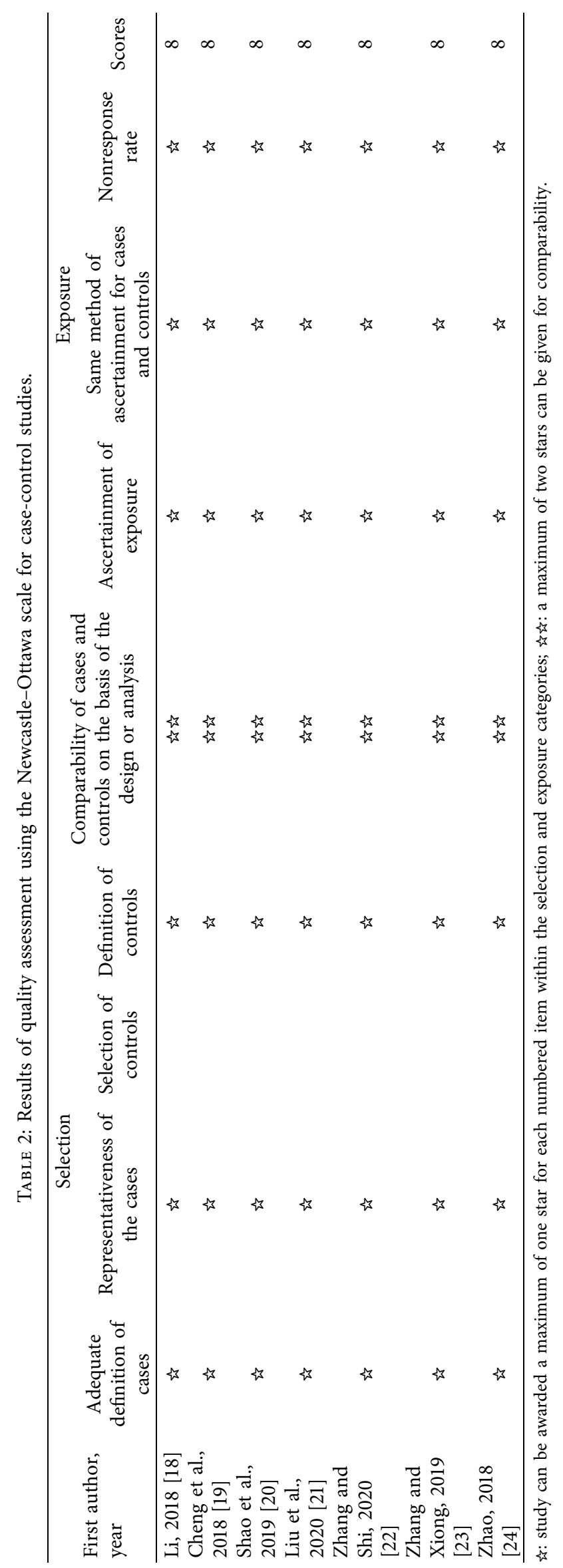




\begin{tabular}{|c|c|c|c|c|c|c|c|c|c|}
\hline \multirow{3}{*}{$\begin{array}{l}\text { Study or Subgroup } \\
\text { Cheng MC } 2018\end{array}$} & \multicolumn{2}{|c|}{ Apatinib } & \multicolumn{2}{|c|}{ Non-Apatinib } & \multirow{2}{*}{$\begin{array}{c}\text { Weight } \\
(\%)\end{array}$} & \multirow{2}{*}{$\begin{array}{l}\text { Odds Ratio } \\
\text { M-H, Fixed, 95\% CI }\end{array}$} & \multirow{2}{*}{\multicolumn{2}{|c|}{$\begin{array}{c}\text { Odds Ratio } \\
\text { M-H, Fixed, } 95 \% \text { CI }\end{array}$}} & \\
\hline & Events & Total & Events & Total & & & & & \\
\hline & 34 & 46 & 16 & 46 & 9.9 & $5.31[2.17,13.00]$ & & $\longrightarrow-$ & \\
\hline Feng Y 2019 & 24 & 30 & 16 & 30 & 7.6 & $3.50[1.11,11.02]$ & & $\longrightarrow$ & \\
\hline Li Q 2018 & 10 & 21 & 4 & 25 & 4.5 & $4.77[1.21,18.78]$ & & $\longrightarrow$ & \\
\hline Liu HM 2020 & 7 & 22 & 4 & 22 & 6.4 & $2.10[0.51,8.57]$ & & $\longrightarrow$ & \\
\hline Quan Y 2020 & 34 & 50 & 24 & 50 & 18.1 & $2.30[1.02,5.19]$ & & $\longrightarrow$ & \\
\hline Ran X 2020 & 15 & 41 & 8 & 41 & 12.0 & $2.38[0.88,6.47]$ & & $\longrightarrow$ & \\
\hline Ren M 2019 & 8 & 38 & 2 & 38 & 3.7 & $4.80[0.95,24.34]$ & & & \\
\hline Shao RX 2019 & 6 & 14 & 4 & 14 & 5.4 & $1.88[0.39,9.01]$ & &. & \\
\hline Wang XY 2019 & 24 & 39 & 21 & 39 & 19.1 & $1.37[0.56,3.38]$ & & $=-$ & \\
\hline Zhang J 2020 & 11 & 21 & 6 & 25 & 6.2 & $3.48[0.99,12.22]$ & & & \\
\hline Zhao LF 2019 & 18 & 19 & 2 & 19 & 0.2 & $153.00[12.68,1845.92]$ & & & $\longrightarrow$ \\
\hline Zhao PP 2018 & 14 & 16 & 10 & 20 & 2.6 & $7.00[1.25,39.15]$ & & _ & \\
\hline Zheng CH 2019 & 37 & 40 & 24 & 40 & 4.3 & $8.22[2.16,31.27]$ & & - & \\
\hline Total (95\% CI) & & 397 & & 409 & 100.0 & $3.51[2.56,4.81]$ & & $\gamma$ & \\
\hline Total events & 242 & & 141 & & & & & & \\
\hline \multicolumn{7}{|c|}{$\begin{array}{l}\text { Heterogeneity: } \mathrm{Tau}^{2}=0.22 ; \mathrm{Chi}^{2}=19.08, \mathrm{df}=12(P=0.09) ; I^{2}=37 \% \\
\text { Test for overall effect: } Z=7.81(P<0.00001)\end{array}$} & $\begin{array}{c}0.1 \\
\text { Apatinib }\end{array}$ & $\begin{array}{lc}1 & 10 \\
\text { Non-Apatinib }\end{array}$ & 1000 \\
\hline
\end{tabular}

Figure 3: Objective response rate of chemotherapy plus apatinib in comparison with chemotherapy alone in the patients with ovarian carcinoma.

\begin{tabular}{|c|c|c|c|c|c|c|c|c|c|c|}
\hline \multirow{3}{*}{$\frac{\text { Study or Subgroup }}{\text { Cheng MC } 2018}$} & \multicolumn{2}{|c|}{ Apatinib } & \multicolumn{2}{|c|}{ Non-Apatinib } & \multirow{2}{*}{$\begin{array}{c}\text { Weight } \\
(\%)\end{array}$} & \multirow{2}{*}{$\begin{array}{c}\text { Odds Ratio } \\
\text { M-H, Fixed, 95\% CI }\end{array}$} & \multirow{2}{*}{\multicolumn{4}{|c|}{$\begin{array}{c}\text { Odds Ratio } \\
\text { M-H, Fixed, 95\% CI }\end{array}$}} \\
\hline & Events & Total & Events & Total & & & & & & \\
\hline & 41 & 46 & 33 & 46 & 10.1 & $3.23[1.04,9.99]$ & & & - & \\
\hline Feng Y 2019 & 29 & 30 & 25 & 30 & 2.4 & $5.80[0.63,53.01]$ & & & & \\
\hline Li Q 2018 & 16 & 21 & 11 & 25 & 6.8 & $4.07[1.14,14.61]$ & & & & \\
\hline Liu HM 2020 & 13 & 22 & 11 & 22 & 12.7 & $1.44[0.44,4.76]$ & & & & \\
\hline Quan Y 2020 & 44 & 50 & 44 & 50 & 14.9 & $1.00[0.30,3.34]$ & & & - & \\
\hline Ran X 2020 & 29 & 41 & 19 & 41 & 15.7 & $2.80[1.13,6.96]$ & & & $\square$ & \\
\hline Ren M 2019 & 21 & 38 & 8 & 38 & 10.1 & $4.63[1.69,12.70]$ & & & & \\
\hline Shao RX 2019 & 9 & 14 & 10 & 14 & 10.1 & $0.72[0.15,3.54]$ & & & - & \\
\hline Wang XY 2019 & 36 & 39 & 29 & 39 & 6.3 & $4.14[1.04,16.44]$ & & & - & \\
\hline Zhang J 2020 & 13 & 21 & 8 & 25 & 7.9 & $3.45[1.02,11.66]$ & & & $\longrightarrow$ & \\
\hline Zhao LF 2019 & 19 & 19 & 10 & 19 & 0.7 & $35.29[1.86,668.20]$ & & & & $\longrightarrow$ \\
\hline Zhao PP 2018 & 16 & 16 & 20 & 20 & & Not estimable & & & & \\
\hline Zheng CH 2019 & 39 & 40 & 33 & 40 & 2.3 & $8.27[0.97,70.73]$ & & & & \\
\hline Total (95\% CI) & & 397 & & 409 & 100.0 & $3.04[2.12,4.36]$ & & & & \\
\hline \multirow{3}{*}{\multicolumn{7}{|c|}{$\begin{array}{l}\text { Heterogeneity: } \mathrm{Chi}^{2}=12.88, \mathrm{df}=11(P=0.30) ; I^{2}=15 \% \\
\text { Test for overall effect: } Z=6.05(P<0.00001)\end{array}$}} & & & & \\
\hline & & & & & & & 0.005 & 0.1 & 10 & 200 \\
\hline & & & & & & & & Apatinib & Non-Apatinib & \\
\hline
\end{tabular}

Figure 4: Disease control rate of chemotherapy plus apatinib in comparison with chemotherapy alone in the patients with ovarian carcinoma.

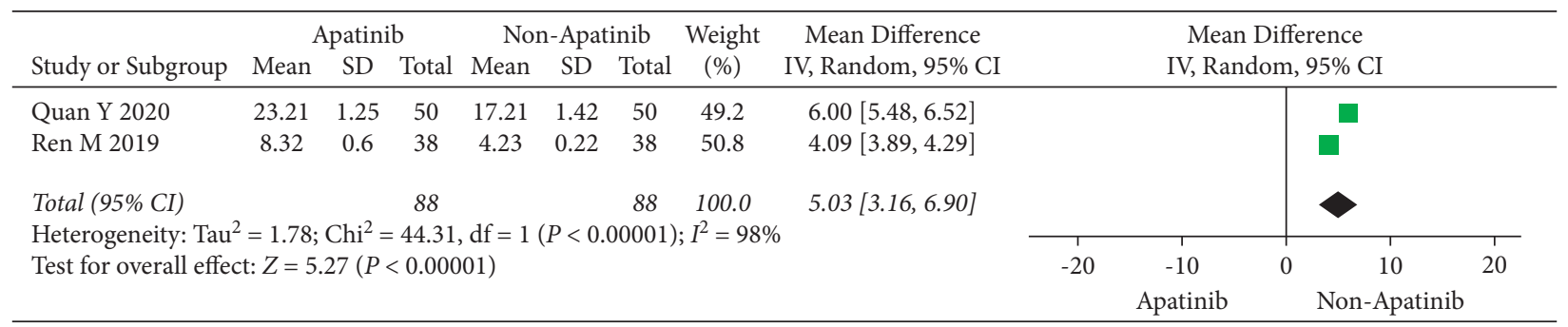

FIGURE 5: Overall survival of chemotherapy plus apatinib in comparison with chemotherapy alone in the patients with ovarian carcinoma. 


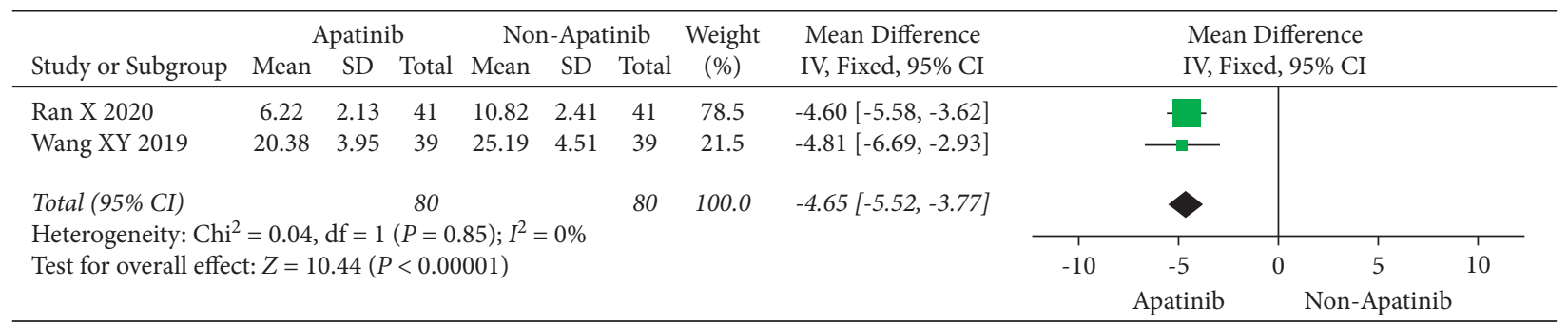

FIGURE 6: The level of CEA chemotherapy plus apatinib in comparison with chemotherapy alone in the patients with ovarian carcinoma.

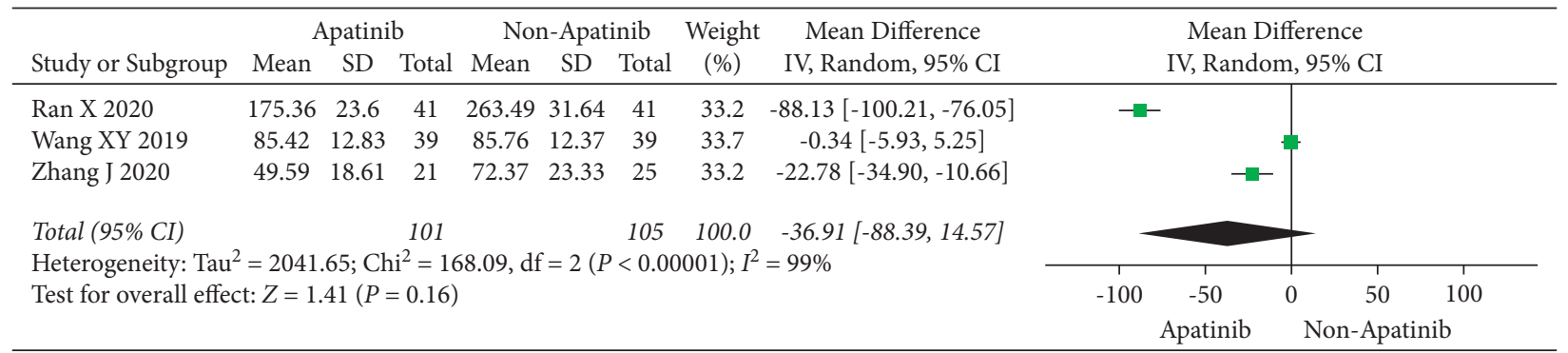

Figure 7: The level of CA125 of chemotherapy plus apatinib in comparison with chemotherapy alone in the patients with ovarian carcinoma.

TABLE 3: Meta-analysis results of adverse drug reactions (Figures S1-S9).

\begin{tabular}{|c|c|c|c|c|c|c|c|c|}
\hline Outcomes & $\begin{array}{c}\text { Number } \\
\text { of } \\
\text { trials }\end{array}$ & $\begin{array}{l}\text { Experimental } \\
\text { group } \\
\text { (events/total) }\end{array}$ & $\begin{array}{c}\text { Control } \\
\text { group } \\
\text { (events/total) }\end{array}$ & SM & OR $(95 \% \mathrm{CI})$ & $I^{2}$ & Heterogeneity & $\begin{array}{c}P \\
\text { value }\end{array}$ \\
\hline Myelosuppression (Figure S1) & 5 & $38 / 162$ & $36 / 166$ & FEM & $1.16(0.61,2.17)$ & $0 \%$ & 0.92 & 0.65 \\
\hline Leucopenia (Figure S2) & 5 & $49 / 119$ & $54 / 127$ & FEM & $1.00(0.56,1.79)$ & $0 \%$ & 0.68 & 0.99 \\
\hline Hypertension (Figure S3) & 11 & $98 / 339$ & $40 / 351$ & REM & $3.18(1.63,6.20)$ & $53 \%$ & 0.02 & $<0.001$ \\
\hline Proteinuria (Figure S4) & 6 & $28 / 148$ & $12 / 160$ & FEM & $3.14(1.51,6.52)$ & $30 \%$ & 0.21 & 0.002 \\
\hline $\begin{array}{l}\text { Gastrointestinal reaction } \\
\text { (Figure S5) }\end{array}$ & 5 & $80 / 165$ & $90 / 173$ & FEM & $0.87(0.55,1.37)$ & $0 \%$ & 0.98 & 0.54 \\
\hline Nausea/vomiting (Figure S6) & 5 & $40 / 161$ & $40 / 161$ & FEM & $1.00(0.58,1.73)$ & $0 \%$ & 0.78 & 1.00 \\
\hline Hand-foot syndrome (Figure S7) & 7 & $60 / 201$ & $19 / 213$ & REM & $\begin{array}{l}4.39(1.59 \\
12.15)\end{array}$ & $55 \%$ & 0.04 & 0.004 \\
\hline $\begin{array}{l}\text { Liver/renal dysfunction } \\
\text { (Figure S8) }\end{array}$ & 4 & $32 / 113$ & $38 / 117$ & FEM & $0.81(0.46,1.44)$ & $0 \%$ & 0.74 & 0.47 \\
\hline Fatigue (Figure S9) & 2 & $8 / 54$ & $7 / 58$ & FEM & $1.23(0.41,3.66)$ & $0 \%$ & 0.48 & 0.71 \\
\hline
\end{tabular}

Note: SM: statistical method, REM: random-effects model, FEM: fixed-effects model, OR: odds ratio, and CI: confidence interval.

apatinib plus chemotherapy who suffered chemotherapy based on taxane plus anthracycline had significantly better ORR $(\mathrm{OR}=8.22 ; 95 \% \mathrm{CI} 2.16,31.27 ; P=0.002)$, and patients receiving apatinib plus platinum-based chemotherapy had considerable DCR $(\mathrm{OR}=4.85 ; 95 \%$ CI 1.94, 12.16; $P<0.001)$.

3.9.6. Chemotherapy Cycle. Less than 4 cycles, 4-6 cycles, more than 6 cycles, and unclear cycles. Subgroup analysis demonstrated that the heterogeneity of the subgroup with patients receiving unclear chemotherapy cycles was more obvious than other subgroups in ORR $\left(I^{2}=55 \% ; P=0.05\right)$ and DCR $\left(I^{2}=51 \% ; P=0.07\right)$, indicating that the subgroup might increase the overall heterogeneity in ORR and DCR. Additionally, subgroup analyses showed that patients receiving apatinib plus chemotherapy who received more than 6 chemotherapy cycles had significantly better ORR $(\mathrm{OR}=8.22 ; 95 \%$ CI 2.16, 31.27; $P=0.002$ ), and patients received apatinib plus chemotherapy who receiving 4-6 chemotherapy cycles had remarkable DCR $(\mathrm{OR}=3.84 ; 95 \%$ CI $1.75,8.45 ; P<0.001)$. 


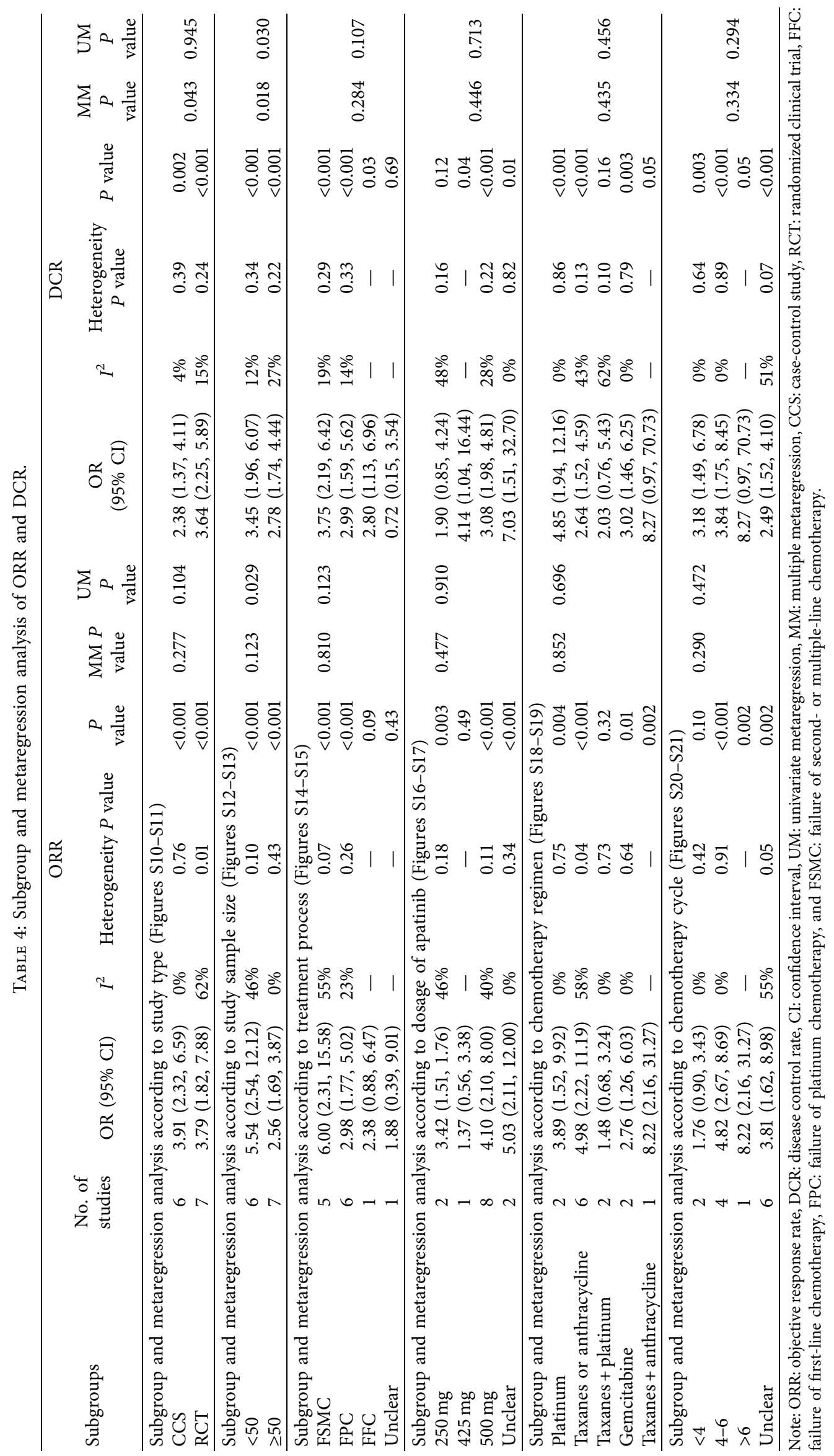


3.10. Publication Bias Analysis. According to the results of funnel plots and Egger/Begg tests, publication bias was shown in these trials for ORR $(P=0.017 ; 95 \%$ CI $0.33,2.67)$, DCR $(P=0.013$; 95\% CI $0.50,3.36)$, and hand-foot syndrome $(P<0.01 ; 95 \% \mathrm{CI}-2.53,-1.65)$, and the other results had no apparent publication bias (Table S1 and Figures S22-S32).

3.11. Sensitivity Analysis. Sensitivity analysis was conducted for ORR, DCR, CA125, myelosuppression, hypertension, proteinuria, leucopenia, gastrointestinal reaction, nausea/ vomiting, hand-foot syndrome, and liver/renal dysfunction by removing the trials one by one. The results of sensitivity analysis demonstrated that except for CA125, most results had good robustness before and after removing trials (Figures S33-S43).

\section{Discussion}

Although OC can be cured in the early stages, once it progresses to an advanced or late stage, few interventions can postpone or stop the malignant illness leading to death. According to the European Society for Medical Oncology and National Comprehensive Cancer Network (NCCN) guidelines for OC [33,34], chemotherapy regimens based on carboplatin plus paclitaxel are the standard treatments for patients with stage II-IV OC. However, many patients relapse after standard treatment and likely develop platinum resistance [35]. There are many available second-line treatment regimens including liposomal doxorubicin, paclitaxel, gemcitabine, etoposide, and so on. Nevertheless, the curative effect of these regimens is extremely limited, of which the objective response rate is less than $30 \%$ and the median progression-free survival (PFS) time is 3 to 4 months $[36,37]$. As no appropriate regimens were available for patients after the second-line treatment regimen [38], OC can easily lead to death once second-line treatment failed.

Angiogenesis has been proved to play an important role in the relapse and metastasis of many cancers such as OC [39] and breast cancer [40]. Vascular endothelial growth factor (VEGF) and its receptor (VEGFR) have been recognized as the critical role of the process of angiogenesis [41]. Over the past decades, many therapeutic agents have been developed targeting to inhibit VEGF and VEGFR, such as bevacizumab and panitumumab [42, 43]. Apatinib, a small molecule inhibitor, can suppress the migration and proliferation of endothelial cells motivated via reducing the tyrosine kinase activity of VEGFR-2 [44]. Ding et al. [45] found that apatinib could suppress tumor growth, migration, and epithelial-mesenchymal transition of OC cells in vivo and in vitro by inhibiting the JAK/STAT3, PI3K/AKT, and Notch signal pathways. Moreover, apatinib has also been proved to promote ROS-dependent apoptosis as well as autophagy in OC cells [46] and inhibit the glycolysis of OC cells via suppressing the VEGFR2/AKT1/SOX5/GLUT4 [47]. Furthermore, apatinib can sensitize resistant tumor cells to chemotherapy drugs and increase the effectiveness of conventional chemotherapy drugs. Tong et al. [48] demonstrated that apatinib could reverse the resistance to docetaxel, daunorubicin, and vincristine in K562/ADR cells. Apatinib has been also proved to improve the antitumor effects of paclitaxel (albumin binding type) in platinum-resistant ovarian cancer cell line and xenograft models [49]. In the past decades, there have been increasing clinical studies on the treatment of OC administrated with apatinib. Chen et al. [50] included 117 eligible patients to evaluate the efficacy and safety of apatinib with a low dose of $250 \mathrm{mg} / \mathrm{d}$ in the treatment of platinum-resistant or platinum-refractory OC patients and then found that the patients administrated with apatinib had satisfactory ORR, DCR, and OS. Miao et al. [51] carried out a phase II study of apatinib and advocated that apatinib $500 \mathrm{mg}$ daily PO is a feasible treatment in patients with recurrent, platinum-resistant, pretreated epithelial ovarian cancer. Additionally, studies have proved that the combination of apatinib and oral etoposide showed promising efficacy and a manageable toxicity profile to treat platinum-resistant or platinum-refractory ovarian cancer $[10,52]$. A real-world study also verified apatinib produced moderate improvements in progression-free survival in patients with platinum-resistant epithelial OC both as maintenance therapy following chemotherapy and as single-agent salvage therapy, and apatinib may be effective for women with platinum-resistant recurrent epithelial ovarian cancer [8].

However, there has been not enough evidence to prove the efficacy and safety of apatinib combined with chemotherapy in the treatment of ovarian carcinoma. To evaluate whether and how the administration of apatinib plus chemotherapy improves clinical efficacy and its safety in patients with ovarian carcinoma who progressed after standard regimens, we included 8 RCTs and 7 CCSs for meta-analysis. Overall, the methodological quality of 8 RCTs and 7 CCSs was found to be medium and high, respectively. This meta-analysis proved that apatinib combined with chemotherapy significantly improved ORR, DCR, and OS and statistically decreased the level of CEA. In relation to adverse drug reactions, apatinib plus chemotherapy increased incidence of hypertension, hand-foot syndrome, and proteinuria while having a similar risk of myelosuppression, leucopenia, gastrointestinal reaction, nausea/vomiting, liver/renal dysfunction, and fatigue when compared to administration with chemotherapy alone. Subgroup and metaregression analysis showed the difference of study type, study sample size, treatment process, dosage of apatinib, chemotherapy regimen, and cycle might increase the overall heterogeneity in ORR and DCR, and some differences were seen between treatment process, dosage of apatinib, chemotherapy regimen, and cycle. Nevertheless, there was slight or no difference among the other subgroups. Publication bias was only shown in these trials for ORR, DCR, and handfoot syndrome. The results of sensitivity analysis demonstrated that most results had good robustness before and after removing trials one by one.

This meta-analysis has several limitations. Firstly, all studies included in our meta-analysis were published in Chinese. We used a comprehensive search strategy and tried to reduce selection bias by searching Cochrane Library, PubMed, Greenmedical, and EBSCO. However, no studies published in English were found. Although we included 15 studies covering 1,020 participants, only two studies included no less than 100 patients. More large-scale randomized double-blind control trials are needed to overcome 
methodological and reporting flaws. In addition, the methods of blinding of all studies were not reported. Most trials had unclear bias risk. Except for the correlation between the study sample size and DCR, univariate and multiple metaregression analysis did not find other positive correlations. In general, all of these limitations might have resulted in an insufficient evaluation of the outcomes.

\section{Conclusion}

In conclusion, most of the included studies showed an unclear risk of bias, and the methodological quality of them was found to be medium and high. Compared with chemotherapy alone, apatinib plus chemotherapy significantly increased ORR, DCR, and OS, with the acceptable risk of adverse drug action. For patients suffering the failure of no less than second-line chemotherapy, $500 \mathrm{mg} /$ day apatinib plus chemotherapy (especially based on taxanes plus anthracycline or platinum for more than 4 cycles) might be the optimal regimen aimed to produce the desired tumor response. Thus, apatinib plus chemotherapy was more effective than chemotherapy alone for the treatment of ovarian carcinoma who progressed after standard regimens. Chemotherapy plus apatinib may provide an additional option for the treatment of suitable patients with the failure of standard regimens. In addition, can apatinib plus chemotherapy improve the long-time survival rate? Can apatinib improve clinical efficacy for drug-resistant patients? Which is the optimal dosage of apatinib and chemotherapy regimen to achieve the best antitumor effect? All these questions need adequately powered and high-quality randomized clinical trials with short- and long-term follow-ups in the future.

\section{Data Availability}

The retrieval strategy was built using MeSH and free words. The retrieval form was (apatinib OR rivoceranib esylate OR YN968D1 OR YN-968D1 OR rivoceranib OR apatinib mesylate) AND ("Ovarian Neoplasms" [Mesh] OR Neoplasm, Ovarian OR Ovarian Neoplasm OR Ovary Neoplasms OR Neoplasm, Ovary OR Neoplasms, Ovary OR Ovary Neoplasm OR Neoplasms, Ovarian OR Ovary Cancer OR Cancer, Ovary OR Cancers, Ovary OR Ovary Cancers OR Ovarian Cancer OR Cancer, Ovarian OR Cancers, Ovarian OR Ovarian Cancers OR Cancer of Ovary OR Cancer of the Ovary). Two investigators independently retrieved all related studies from the following databases: China Biological Medicine Database (CBM), China National Knowledge Infrastructure Database (CNKI), Chinese Scientific Journals Full-Text Database (VIP), Wanfang Data, Cochrane Library, PubMed, Greenmedical, and EBSCO (up to October 2020).

\section{Conflicts of Interest}

The authors declare that they have no conflicts of interest.

\section{Acknowledgments}

This study was supported by the Natural Science Foundation of Jiangsu Province for Youths (No. BK20180277), China
Postdoctoral Science Foundation (No. 137070586), and the Second Traditional Chinese Medicine Leadership Training Foundation of Jiangsu Province (Clinical Study on Prevention and Treatment of Postoperative Recurrence and Metastasis of Gastric Cancer Based on "Fuzheng Qingdu" Syndrome).

\section{Supplementary Materials}

Supplementary Material 1. Figures S1-S21: forest plots of subgroup and metaregression analysis; Supplementary Material 2. Table S1 and Figures S22-32: results of publication bias analysis; and Supplementary Material 3. Figures S33-43: results of sensitivity analysis. (Supplementary Materials)

\section{References}

[1] F. Bray, J. Ferlay, I. Soerjomataram, R. L. Siegel, L. A. Torre, and A. Jemal, "Global cancer statistics 2018: GLOBOCAN estimates of incidence and mortality worldwide for 36 cancers in 185 countries," CA: A Cancer Journal for Clinicians, vol. 68, no. 6, pp. 394-424, 2018.

[2] B. A. Werness and G. H. Eltabbakh, "Familial ovarian cancer and early ovarian cancer: biologic, pathologic, and clinical features," International Journal of Gynecological Pathology, vol. 20, no. 1, pp. 48-63, 2001.

[3] G. C. Jayson, E. C. Kohn, H. C. Kitchener, and J. A. Ledermann, "Ovarian cancer," The Lancet, vol. 384, no. 9951, pp. 1376-1388, 2014.

[4] C. Liu, H. Pan, P. Torng, M. Fan, and T. Mao, "SRPX and HMCN1 regulate cancer-associated fibroblasts to promote the invasiveness of ovarian carcinoma," Oncology Reports, vol. 42, no. 6, pp. 2706-2715, 2019.

[5] R. A. Burger, "Overview of anti-angiogenic agents in development for ovarian cancer," Gynecologic Oncology, vol. 121, no. 1, pp. 230-238, 2011.

[6] Y. Xu, Z. Huang, H. Lu et al., "Apatinib in patients with extensive-stage small-cell lung cancer after second-line or third-line chemotherapy: a phase II, single-arm, multicentre, prospective study," British Journal of Cancer, vol. 121, no. 8, pp. 640-646, 2019.

[7] Z. Liu, J. Chen, Y. Fang, X. Han, H. Pan, and W. Han, "The efficacy and safety of apatinib treatment for patients with unresectable or relapsed liver cancer: a retrospective study," Journal of Cancer, vol. 9, no. 16, pp. 2773-2777, 2018.

[8] J. Zhang, A. Li, Q. Jiang, F. Zheng, and H. Zhu, "Efficacy and safety of apatinib treatment in platinum-resistant recurrent epithelial ovarian cancer: a real world study," Drug Design, Development and Therapy, vol. 13, no. 13, pp. 3913-3918, 2019.

[9] M. Yang, X. Liu, C. Zhang et al., "A study of efficacy and safety with apatinib or apatinib combined with chemotherapy in recurrent/advanced ovarian cancer patients," Cancer Management and Research, vol. 11, pp. 8869-8876, 2019.

[10] Q. Huang, C. Chu, J. Tang, and Z. Dai, "Efficacy and safety of apatinib combined with etoposide in patients with recurrent platinum-resistant epithelial ovarian cancer: a retrospective study," Journal of Cancer, vol. 11, no. 18, pp. 5353-5358, 2020.

[11] H. Watanabe, S. Yamamoto, H. Kunitoh et al., "Tumor response to chemotherapy: the validity and reproducibility of RECIST guidelines in NSCLC patients1," Cancer Science, vol. 94, no. 11, pp. 1015-1020, 2003. 
[12] A. B. Miller, B. Hoogstraten, M. Staquet, and A. Winkler, "Reporting results of cancer treatment," Cancer, vol. 47, no. 1, pp. 207-214, 1981.

[13] A. Trotti, A. Colevas, A. Setser et al., "CTCAE v3.0: development of a comprehensive grading system for the adverse effects of cancer treatment," Seminars in Radiation Oncology, vol. 13, no. 3, pp. 176-181, 2003.

[14] P. Guyot, A. E. Ades, M. J. Ouwens, and N. J. Welton, "Enhanced secondary analysis of survival data: reconstructing the data from published Kaplan-Meier survival curves," $B M C$ Medical Research Methodology, vol. 12, p. 9, 2012.

[15] Z. Xiao, Y. Jiang, C.-Q. Wang et al., "Clinical efficacy and safety of aidi injection combination with vinorelbine and cisplatin for advanced non-small-cell lung carcinoma: a systematic review and meta-analysis of 54 randomized controlled trials," Pharmacological Research, vol. 153, Article ID 104637, 2020.

[16] M. Egger, G. D. Smith, M. Schneider, and C. Minder, "Bias in meta-analysis detected by a simple, graphical test," $B M J$, vol. 315, no. 7109, pp. 629-634, 1997.

[17] X. Sun, M. Briel, S. D. Walter, and G. H. Guyatt, "Is a subgroup effect believable? Updating criteria to evaluate the credibility of subgroup analyses," BMJ, vol. 340, no. mar30 3, p. c117, 2010.

[18] Q. Li, Clinical Observation of Mesylate Tablets Combined Chemotherapy for Advanced Ovarian Cancer, Hebei North University, Zhangiiakou, China, 2018.

[19] M. Cheng, S. Xu, H. Yao, J. Liu, G. Sun, and T. Liu, "Efficacy and safety of low-dose apatinib combined with docetaxel versus docetaxel in the treatment of ovarian cancer with second-line or above chemotherapy," Guide of China Medicine, vol. 16, no. 13, pp. 203-204, 2018.

[20] R. Shao, L. Han, x. Chen, and H. Wang, "Clinical efficacy and safety of apatinib combined with TP in the treatment of recurrent ovarian cancer after the failure of platinum-based chemotherapy," Electronic Journal of Practical Gynecologic Endocrinology, vol. 6, no. 34, pp. 124-125, 2019.

[21] H. Liu, D. Cui, and Y. Li, "Clinical efficacy of apatinib combined with chemotherapy in the treatment of late ovarian cancer with the failure of second-line chemotherapy," Chinese Journal of Clinical Oncology and Rehabilitation, vol. 27, no. 3, pp. 316-318, 2020.

[22] J. Zhang and H. Shi, "Short-term efficacy and safety of apatinib in the treatment of platinum-resistant ovarian cancer," International Journal of Gynecology, vol. 47, no. 2, pp. 152-154, 2020.

[23] H. Zhang and Z. Xiong, "Efficacy of apatinib combined with cisplatin peritoneal infusion in the treatment of malignant peritoneal effusion of ovarian Cancer," Chinese and Foreign Medical Research, vol. 17, no. 21, pp. 139-141, 2019.

[24] P. Zhao, The Short-Term Efficacy and Safety of Combined Apatinib and Liposomal Doxorubicin in the Treatment of Recurrent Ovarian Cancer, Zhengzhou University, Zhengzhou, China, 2018.

[25] Y. Feng, Y. Wan, X. Wang, and C. Jiang, "Clinical effect of apatinib combined with chemotherapy on patients with drugresistant ovarian cancer," Clinical Research and Practice, vol. 33, pp. 20-21, 2019.

[26] M. Ren, "Clinical efficacy of apatinib combined with intraperitoneal injection of cisplatin in treatment of elderly advanced ovarian cancer patients with malignant ascites," Modern Medicine Journal of China, vol. 21, no. 12, pp. 9-12, 2019.
[27] X. Wang and F. Qu, "Clinical study on apatinib combined with DP regime in treatment of advanced ovarian cancer," Drugs \& Clinic, vol. 34, no. 7, pp. 2132-2136, 2019.

[28] Y. Liu, "Efficacy and safety of Apatinib in the treatment of ovarian cancer," Clinical Research, vol. 23, no. 5, p. 76, 2015.

[29] L. Zhao, C. Wu, and D. Hou, "Clinical analysis of apatinib combined with chemotherapy for second-line failed advanced ovarian cancer," Electronic Journal of Clinical Medical Literature, vol. 6, no. 67, p. 171, 2019.

[30] C. Zheng, Z. Tang, and T. Liu, "Discussion on efficacy of apatinib combined with chemotherapy in the treatment of recurrence of epithelial ovarian cancer," Chinese Journal of Modern Drug Application, vol. 13, no. 23, pp. 106-108, 2019.

[31] Y. Quan, "Efficacy of apatinib combined with chemotherapy in the treatment of drug-resistant ovarian cancer," China Practical Medical, vol. 15, no. 11, pp. 117-118, 2020.

[32] X. Ran and X. Liu, "Evaluation of the efficacy and safety of Apatinib combined with GEMOX regimen for advanced refractory," Chinese Journal of Human Sexuality, vol. 29, no. 1, pp. 44-48, 2020.

[33] R. J. Morgan, D. K. Armstrong, R. D. Alvarez et al., "Ovarian cancer, version 1.2016, NCCN clinical practice guidelines in Oncology," Journal of the National Comprehensive Cancer Network: Journal of the National Comprehensive Cancer Network, vol. 14, no. 9, pp. 1134-1163, 2016.

[34] J. A. Ledermann, F. A. Raja, C. Fotopoulou, A. GonzalezMartin, N. Colombo, and C. Sessa, "Corrections to "Newly diagnosed and relapsed epithelial ovarian carcinoma: ESMO clinical practice guidelines for diagnosis, treatment and follow-up"," Annals of Oncology, vol. 29, no. Suppl 4, p. iv259, 2018.

[35] M. Champer, Y. Huang, J. Y. Hou et al., "Adherence to treatment recommendations and outcomes for women with ovarian cancer at first recurrence," Gynecologic Oncology, vol. 148, no. 1, pp. 19-27, 2018.

[36] G. Corrado, V. Salutari, E. Palluzzi, M. G. Distefano, G. Scambia, and G. Ferrandina, "Optimizing treatment in recurrent epithelial ovarian cancer," Expert Review of Anticancer Therapy, vol. 17, no. 12, pp. 1147-1158, 2017.

[37] E. Pujade-Lauraine, F. Hilpert, B. Weber et al., "Bevacizumab combined with chemotherapy for platinum-resistant recurrent ovarian cancer: the AURELIA open-label randomized phase III trial," Journal of Clinical Oncology, vol. 32, no. 13, pp. 1302-1308, 2014.

[38] B. J. Monk, L. M. Randall, and R. N. Grisham, “The evolving landscape of chemotherapy in newly diagnosed advanced epithelial ovarian cancer," American Society of Clinical Oncology Educational Book, vol. 39, no. 39, pp. e141-e151, 2019.

[39] M. Akbarzadeh, A. A. Movassaghpour, H. Ghanbari et al., "The potential therapeutic effect of melatonin on human ovarian cancer by inhibition of invasion and migration of cancer stem cells," Scientific Reports, vol. 7, no. 1, p. 17062, 2017.

[40] S. Soheilyfar, Z. Velashjerdi, Y. Sayed Hajizadeh et al., "In vivo and in vitro impact of miR-31 and miR-143 on the suppression of metastasis and invasion in breast cancer," Journal of BUON: Official Journal of the Balkan Union of Oncology, vol. 23, no. 5, pp. 1290-1296, 2018.

[41] P. Carmeliet, "VEGF as a key mediator of angiogenesis in cancer," Oncology, vol. 69, no. Suppl 3, pp. 4-10, 2005.

[42] M. Arjaans, C. P. Schröder, S. F. Oosting, U. Dafni, J. E. Kleibeuker, and E. G. E. De Vries, "VEGF pathway targeting agents, vessel normalization and tumor drug uptake: 
from bench to bedside," Oncotarget, vol. 7, no. 16, pp. 21247-21258, 2016.

[43] S. Heskamp, O. C. Boerman, J. D. M. Molkenboer-Kuenen, W. J. G. Oyen, W. T. A. van der Graaf, and H. W. M. Van Laarhoven, "Bevacizumab reduces tumor targeting of antiepidermal growth factor and anti-insulin-like growth factor 1 receptor antibodies," International Journal of Cancer, vol. 133, no. 2, pp. 307-314, 2013.

[44] Y. Wang, M. Deng, Q. Chen et al., "Apatinib exerts anti-tumor activity to non-Hodgkin lymphoma by inhibition of the Ras pathway," European Journal of Pharmacology, vol. 843, pp. 145-153, 2019.

[45] J. Ding, X.-Y. Cheng, S. Liu et al., "Apatinib exerts anti-tumour effects on ovarian cancer cells," Gynecologic Oncology, vol. 153, no. 1, pp. 165-174, 2019.

[46] X. Sun, J. Li, Y. Li, S. Wang, and Q. Li, “Apatinib, a novel tyrosine kinase inhibitor, promotes ROS-dependent apoptosis and autophagy via the Nrf2/HO-1 pathway in ovarian cancer cells," Oxidative Medicine and Cellular Longevity, vol. 2020, Article ID 3145182, 19 pages, 2020.

[47] L. Chen, X. Cheng, W. Tu et al., "Apatinib inhibits glycolysis by suppressing the VEGFR2/AKT1/SOX5/GLUT4 signaling pathway in ovarian cancer cells," Cellular Oncology, vol. 42, no. 5, pp. 679-690, 2019.

[48] X.-Z. Tong, F. Wang, S. Liang et al., "Apatinib (YN968D1) enhances the efficacy of conventional chemotherapeutical drugs in side population cells and ABCB1-overexpressing leukemia cells," Biochemical Pharmacology, vol. 83, no. 5, pp. 586-597, 2012.

[49] H. Zhao, R. Li, X. Wang et al., "Retracted article: the role of apatinib combined with paclitaxel (aluminum binding type) in platinum-resistant ovarian cancer," Journal of Ovarian Research, vol. 13, no. 1, p. 113, 2020.

[50] W. Chen, Z. Li, Z. Zheng, and X. Wu, "Efficacy and safety of low-dose apatinib in ovarian cancer patients with platinumresistance or platinum-refractoriness: a single-center retrospective study," Cancer medicine, vol. 9, no. 16, pp. 5899-5907, 2020.

[51] M. Miao, G. Deng, S. Luo et al., "A phase II study of apatinib in patients with recurrent epithelial ovarian cancer," Gynecologic Oncology, vol. 148, no. 2, pp. 286-290, 2018.

[52] C.-Y. Lan, Y. Wang, Y. Xiong et al., "Apatinib combined with oral etoposide in patients with platinum-resistant or platinum-refractory ovarian cancer (AEROC): a phase 2, singlearm, prospective study," The Lancet Oncology, vol. 19, no. 9, pp. 1239-1246, 2018. 